\title{
AN IDEALIZED MODEL OF TIDAL DYNAMICS IN SEMI-ENCLOSED BASINS: THE EFFECTS OF A MEGA-SCALE SAND EXTRACTION TRENCH IN THE NORTH SEA
}

\author{
Wiebe P. de Boer ${ }^{1,2}$, Pieter C. Roos ${ }^{1}$, Suzanne J.M.H. Hulscher ${ }^{1}$ and Ad Stolk
}

\begin{abstract}
We investigate the effects of a mega-scale sand extraction trench (length $\sim 200 \mathrm{~km}$, width $\sim 10 \mathrm{~km}$, depth $\sim 10 \mathrm{~m}$, located in front of the Dutch coast) on tidal dynamics in the Southern North Sea. To this end, an idealized modeling approach is adopted, extending the classical problem of Kelvin wave reflection in rectangular semi-enclosed basins to account for lateral depth variations and the presence of a trench. The model results indicate changes in tidal range (zones of decrease and increase of the order of several $\mathrm{cm}$ ), phase and currents (order $\mathrm{cm} / \mathrm{s}$ ) throughout the whole basin, which emphasizes the importance of a model domain on the scale of the basin. The changes in currents may structurally change sediment transport rates by several percents, which is relevant to coastal morphology. It is argued that this idealized model can be used to support a companion study carried out with a more detailed numerical engineering model. This is because of its ability to (1) provide insight into the physical mechanisms, (2) reveal the order of magnitude and area of influence of the effects and (3) allow for a quick sensitivity analysis with respect to trench geometry.
\end{abstract}

Keywords: sand extraction, semi-enclosed basins, tidal dynamics, North Sea, Netherlands Continental Shelf

\section{INTRODUCTION}

Sand extraction is one of the most important engineering activities in the North Sea, particularly on the Netherlands Continental Shelf (NCS). Sand is extracted from a designated area on the NCS, between the established NAP -20 m depth contour (NAP is the Dutch Ordnance Level) and the 12 miles boundary. In the future sand extraction from the NCS is expected to increase considerably, mainly due to increasing nourishment volumes that are required to cope with expected sea level rise. Consequently, sand extraction volumes are expected to increase from $25 \times 10^{6} \mathrm{~m}^{3}$ per year at present to $60 \times 10^{6} \mathrm{~m}^{3}$ or even $110 \times 10^{6} \mathrm{~m}^{3}$ per year in the next decades. On the long term (over decades to centuries), this repeated and increasing sand extraction makes the gradual creation of a continuous extraction trench along the entire Dutch coast a possible scenario. This scenario is currently considered by Rijkswaterstaat Noordzee (part of the Netherlands Ministry of Transport, Public Works and Water Management), responsible for developing a long-term extraction strategy on the NCS. Such a 'megascale' trench (length hundreds of km, width over $10 \mathrm{~km}$, depth several $\mathrm{m}$ ) implies extraction on a much larger scale than in current practice (termed 'large-scale'). Therefore, its impacts on the hydrodynamic, morphodynamic and ecological system of the North Sea are currently unknown.

In this study we focus on the impact of such a mega-scale trench on tidal dynamics, a key aspect in hydrodynamics and indirectly also affecting morphodynamics and ecology. What are the effects of a mega-scale trench on the so-called elevation and current amphidromic system, i.e. the spatial pattern of tidal ranges and currents? And how do these changes depend on the trench geometry?

To answer these questions, two modeling approaches can be followed: (1) engineering and (2) idealized models. In engineering practice, process-based numerical simulation models (e.g., Delft3D, MIKE21, Telemac) are often used. This type of models, characterized by detailed model geometries and state-of-the-art formulations of many physical processes, is particularly helpful in answering sitespecific engineering questions. However, simulations usually require a rather large computational effort, which makes it difficult to conduct extensive sensitivity analyses and to obtain insight in physical mechanisms. Alternatively, idealized process-based models are specifically designed to achieve such generic insight. Typical properties are the schematizations of geometry and physics, attempting to retain only the essential features, processes and mechanisms. Moreover, emphasis is put on the transparency of (semi-)analytical solution techniques. As a result, idealized models are usually quick to run, thus allowing for extensive sensitivity analyses with respect to geometry and physics.

In this study, we follow the idealized modeling approach to investigate the impact of a mega-scale trench on tidal dynamics in the North Sea (see figure 1). In the remainder of this paper, we introduce

\footnotetext{
${ }^{1}$ Water Engineering and Management, Faculty of Engineering Technology, University of Twente, P.O. Box 217, 7500 AE Enschede, The Netherlands

${ }^{2}$ Harbour, Coastal and Offshore Engineering; Unit Hydraulic Engineering, Deltares, P.O. Box 177, 2600 MH Delft, The Netherlands

${ }^{3}$ Ministry of Infrastructure and the Environment, Rijkswaterstaat Noordzee, P.O. Box 5807, 2280 HV Rijswijk, The Netherlands
} 


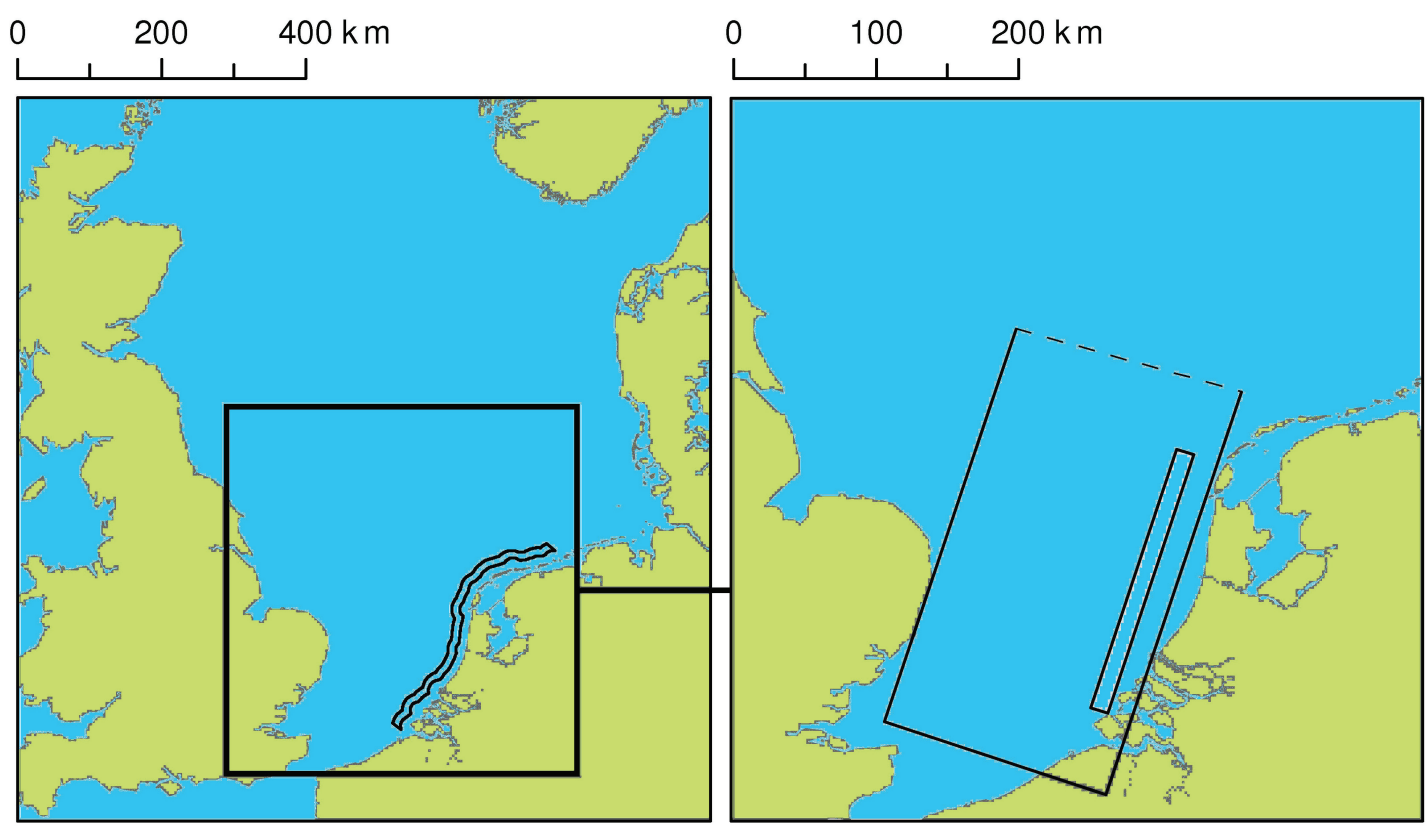

Figure 1. Left: map of the North Sea with a mega-scale sand extraction trench in front of the Dutch coast. Right: schematized representation of the Southern North Sea and the trench in our idealized model.

the hydrodynamic model, describe and discuss the main results and present our conclusions. We note that, parallel to our study, Van der Werf et al. (2011) investigated the same problem using a typical engineering model.

\section{IDEALIZED HYDRODYNAMIC MODEL}

\section{Background and model set-up}

Our idealized hydrodynamic model extends Taylor's (1922) classical model of Kelvin wave reflection in a semi-enclosed, rotating basin of uniform depth and width. Taylor's solution, satisfying the inviscid depth-averaged linear shallow water equations on the $f$ plane, can be written as a superposition of eigenmodes: an incoming Kelvin wave (which forces the problem), an infinite set of Poincaré modes (generated at the basin's closed end) and a reflected Kelvin wave (propagating out of the basin). These eigenmodes are fundamental wave solutions in a rotating, open channel of uniform depth and width; see e.g. Gill (1982).

Despite the strong schematizations, Taylor's model qualitatively reproduces and explains the main features of amphidromic systems in tidal basins such as the North Sea. These features include the presence and positioning of elevation and current amphidromic points (EAPs, CAPs), where elevation fluctuations and velocity amplitudes are zero, respectively and the elliptical shape of the particle trajectories near the basins's closed end. Extending Taylor's model to account for dissipation, Rienecker \& Teubner (1980) showed that incorporating a linear bottom friction law leads to damping of the Kelvin waves as they propagate. This causes shifts in the EAPs and CAPs and thus affects the details of the amphidromic system. The ability of this type of models to actually reproduce tide observations in semi-enclosed basins has been shown recently by Roos \& Schuttelaars (2011).

To represent a mega-scale trench in the Southern North Sea, our idealized model extends Rienecker \& Teubner's (1980) frictional version of Taylor's (1922) model in the following two respects (see figures 1 and 2).

- Inclusion of lateral depth profiles $h(x)$ with cross-basin coordinate $x$, for which a typical depth profile of the Southern North Sea profile is taken (figure $2 a$ ).

- Distinction in three adjacent compartments $\mathrm{C} 1-\mathrm{C} 3$, the middle one (C2, of length $\left.L_{\mathrm{tr}}\right)$ containing the trench as a local shore-parallel deepening of width $B_{\text {tr }}$ (figure $2 \mathrm{~b}$ ).

The basin width, length and mean depth are given by $B=157 \mathrm{~km}$ and $L=600 \mathrm{~km}$ and $H_{0}=29.2 \mathrm{~m}$, respectively. These values are based on bathymetric data from the Southern North Sea, so is the depth profile $h(x)$, e.g. see Van der Veen \& Hulscher (2009). This profile, shown in figure 2a, describes the 


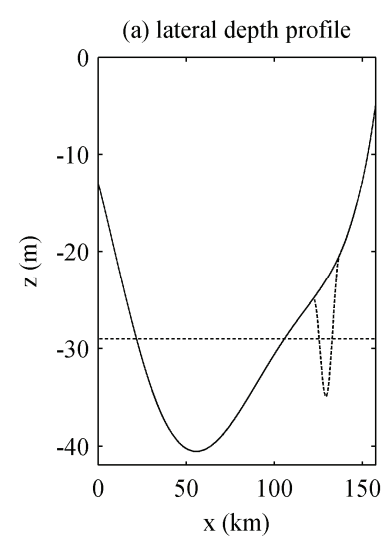

(b) geometry

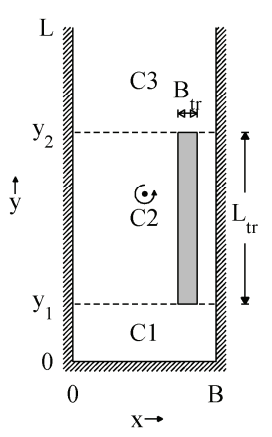

(c) solution method

(d) indicators

Figure 2. (a) Typical lateral depth profile $z=-h(x)$ for the Southern North Sea (solid), the sinusoidal trench shape with $B_{\mathrm{tr}}=15 \mathrm{~km}$ and $H_{\mathrm{tr}}=12 \mathrm{~m}$ (dashed) and the mean water depth (dotted). (b) Top view sketch of the model geometry, showing a semi-enclosed basin with three compartments $\mathrm{C} 1-\mathrm{C} 3$, the middle one containing a rectangular trench of length $L_{\mathrm{tr}}$ and width $B_{\mathrm{tr}}$ (grey shade). (c) Sketch of the solution method, showing the collocation points (black circles) and the sets of Kelvin and Poincaré modes (double arrows) and the incoming Kelvin wave (single arrow). (d) For interpretation of the results, the coastline is divided into seven sections S1-S7; the trench center is denoted by $Q$.

depth in all compartments $\mathrm{C} 1-\mathrm{C} 3$, be it that compartment $\mathrm{C} 2$ - used to represent the trench - contains the trench as a further local deepening. The trench has length $L_{\mathrm{tr}}$, width $B_{\mathrm{tr}}$ (figure $2 \mathrm{~b}$ ) and a sinusoidal shape with maximum depth $H_{\text {tr }}$, such that the total volume of sand represented by the trench is given by

$$
V_{\mathrm{tr}}=1 / 2 H_{\mathrm{tr}} B_{\mathrm{tr}} L_{\mathrm{tr}} .
$$

Note that along-basin depth variations inside the compartments are neglected.

\section{Model equations and boundary/matching conditions}

We consider a semi-diurnal lunar tidal wave (the M2-tide) of angular frequency $\sigma=1.41 \times 10^{-4} \mathrm{rad} / \mathrm{s}$ and typical elevation amplitude $A \sim 1.5 \mathrm{~m}$, propagating through a rectangular semi-enclosed basin with dimensions as introduced above. Closed basin boundaries are located at $x=0, x=B$ and $y=0$, the open basin boundary at $y=L$ (see figure $2 \mathrm{~b}$ ).

Assuming that $A / H_{0}$ is small, we express conservation of mass and momentum by the linear depthaveraged shallow water equations on the $f$-plane, with a linear bottom friction law, i.e.

$$
\begin{aligned}
& u_{t}-f v+r u / h=-g \zeta_{x}, \\
& v_{t}+f u+r v / h=-g \zeta_{y}, \\
& \zeta_{t}+(h u)_{x}+h v_{y}=0 .
\end{aligned}
$$

Here, $u$ and $v$ are the components of the depth-averaged flow velocity vector in the horizontal $x$ and $y$ directions, respectively, $\zeta$ the free surface elevation and $h$ the local depth. Furthermore, $f=2 \Omega \sin \theta$ is a Coriolis parameter (with angular frequency of the Earth's rotation $\Omega=7.292 \times 10^{-5} \mathrm{rad} / \mathrm{s}$ and latitude $\left.\theta=52.5^{\circ} \mathrm{N}\right), g=9.81 \mathrm{~m} / \mathrm{s}^{2}$ the gravitational acceleration and $r=8 C_{D} U /(3 \pi)$ a linear friction parameter with drag coefficient $C_{D}=2.5 \times 10^{-3}$ and typical velocity scale $U$. This velocity scale is estimated from a classical Kelvin wave propagating over a uniform depth $H_{0}$ without bottom friction: $U=A\left(g / H_{0}\right)^{1 / 2} \sim 0.87$ $\mathrm{m} / \mathrm{s}$. Finally, in the formulation of the continuity equation (2c), we have used the fact that we consider cross-basin depth variations only, i.e. $h_{y}=0$.

At the closed boundaries of the basin, we impose a no-normal flow condition, i.e.

$$
\begin{gathered}
u=0 \quad \text { at } x=0 \text { and } x=B, \\
v=0 \quad \text { at } y=0 .
\end{gathered}
$$

Next, we require the free surface elevation and the along-basin mass flux to be continuous across the interfaces $y=y_{1}$ (between $\mathrm{C} 1$ and C2) and $y=y_{2}$ (between C2 and C3):

$$
\zeta_{1}=\zeta_{2} \text { and }(h v)_{1}=(h v)_{2} \text { at } y=y_{1} .
$$




$$
\zeta_{2}=\zeta_{3} \text { and }(h v)_{2}=(h v)_{3} \quad \text { at } y=y_{2} \text {. }
$$

Here, subscripts 1, 2 and 3 denote the solutions in the three compartments C1-C3, respectively. Finally, analogous to Taylor (1922) and Rienecker \& Teubner (1980), the problem is forced by an incoming Kelvin wave with coastal amplitude $A$ at $x=L$, while allowing reflected Kelvin and Poincaré waves to leave the domain.

\section{Solution method}

The solution method is analogous to the literature (e.g., Rienecker \& Teubner 1980) in the sense that we seek time-periodic solutions written as a truncated superposition of eigenmodes and adopt a collocation technique to obtain the coefficients of each mode. However, three complications arise, which will be briefly explained below. An extensive description of the solution method is given by De Boer et al. (submitted).

The first complication with respect to Rienecker \& Teubner (1980) is that we must distinguish a solution in each of the three compartments $\mathrm{C} 1-\mathrm{C} 3$, i.e.

$$
\zeta_{j}(x, y)=\operatorname{Re}\left\{S_{j}(x, y) \exp (-i \sigma t)\right\} .
$$

Here, Re denotes the real part and $S_{j}(x, y)$ represents the (complex) amplitude of the free surface elevation in compartment $j=1,2,3$. Similar expressions define the complex amplitudes $U_{j}$ and $V_{j}$ of the flow field in compartment $j$.

The second complication is that, due to the cross-basin variations in the depth-profile $h(x)$, the cross-basin structure of the eigenmodes is no longer available analytically. The along-basin depth uniformity still warrants a harmonic along-basin structure, i.e. wave solutions are of a form $S(x, y)=\hat{S}(x) \exp (i k y)$, with cross-basin structure $\hat{S}(x)$ and wave number $k$. The (complex) wave number is obtained iteratively, starting with the $k$-value from Rienecker $\&$ Teubner's (1980) analysis for a uniform depth $H_{0}$. For a given $k$-value, the cross-basin wave structure $\hat{S}(x)$ satisfies a second order linear boundary value problem with variable coefficients, which is solved numerically using a fourth order Runge-Kutta (RK4) method. The fact that each mode must satisfy the no-normal flow condition (3a) at both channel boundaries $y=0$ and $y=B$ is then used to iterate and improve the estimate of the wave number $k$. As it turns out, this iterative procedure converges to give us both the wave numbers and the cross-basin structures of the Kelvin and Poincaré modes for our typical North Sea profile, with or without the superimposed trench profile. Note that, due to the asymmetric profile, the modes propagating (if free) or exponentially decaying (if evanescent) in the positive and negative $y$-direction are different.

The third complication deals with the collocation technique, which must be extended to account not only for the no-normal flow condition ( $3 b$ ) at the basin's closed end, but also for the matching conditions (4) at the interfaces between the compartments (figure 2c). From a physical perspective, the topographic steps at the trench edges $y=y_{1}$ and $y=y_{2}$ affect the way in which Kelvin modes are reflected/transmitted, thus triggering two series of Poincaré modes per interface, bound to either side of the interface. This can be seen from the double arrows in figure 2c. Mathematically, with truncation number $M$ (denoting the highest Poincaré mode involved in each of the Poincaré series depicted in figure 2c), we now obtain a linear set of $5(M+1)$ equations with the $5(M+1)$ coefficients as unknowns, which - just like the system of dimension $M+1$ in e.g. Rienecker \& Teubner (1980) - is solved using standard techniques.

\section{MODEL RESULTS}

\section{Effects of trench on tidal dynamics}

To investigate the effects of a mega-scale trench on the tidal dynamics in the North Sea, we introduce a reference trench with dimensions $L_{\mathrm{tr}}=224 \mathrm{~km}, B_{\mathrm{tr}}=15 \mathrm{~km}$ and $H_{\mathrm{tr}}=12 \mathrm{~m}$ and, according to equation (1), an extraction volume of $V_{\mathrm{tr}}=20.2 \times 10^{9} \mathrm{~m}^{3}$. In all of our simulations, the truncation number is set at $M=80$.

The model results for this reference situation are depicted in figures 3, 4 and 5, showing tidal ranges, along-basin flow velocities and cross-basin flow velocities, respectively. Each of these figure shows (a) the situation without trench, (b) the situation with the reference trench and (c) the differences between these two situations. Herein, positive values and red shades indicate increases due to the trench, whereas negative values and blue shades indicate decreases due to the trench. Furthermore, the squares and circles denote the coastal locations of maximum increase and decrease, evaluated for each of the seven coastal sections S1-S7 introduced previously in figure 2d. We note that the elevation and 
(a) without trench [m]

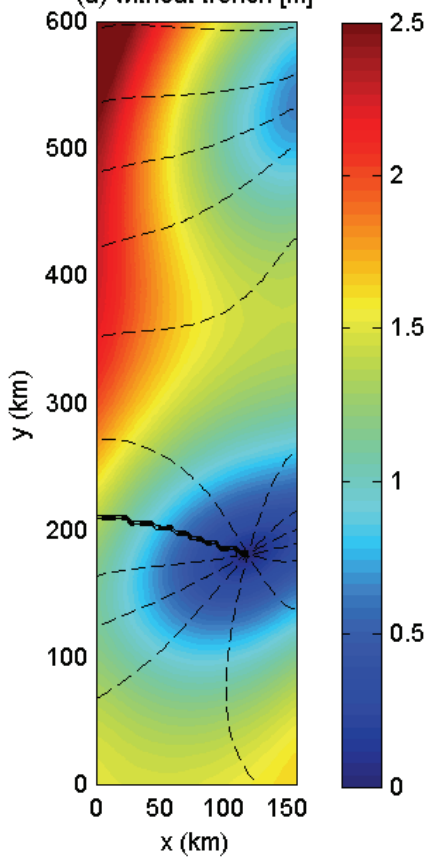

(b) with ref. trench $[\mathrm{m}]$

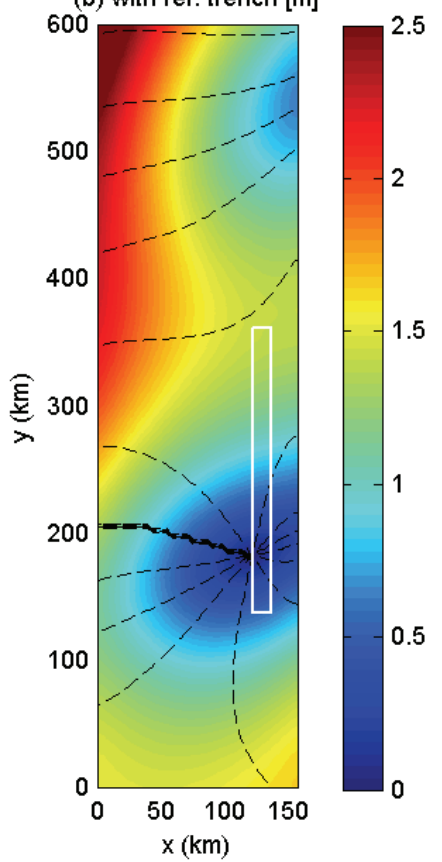

(c) differences $[\mathrm{cm}]$

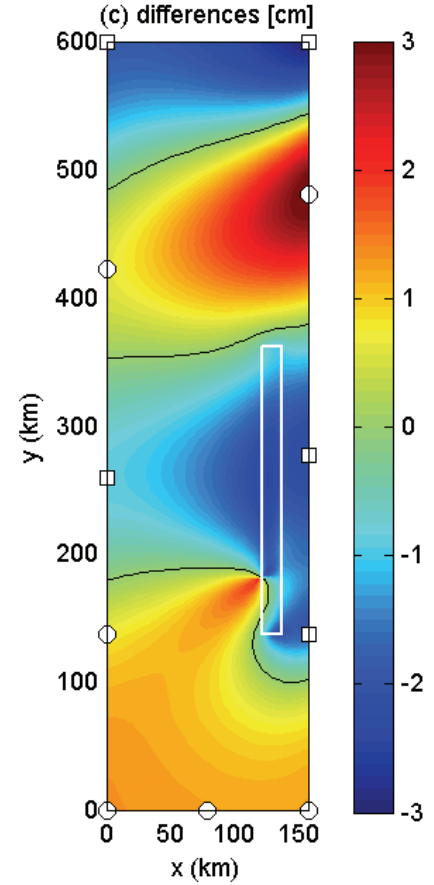

Figure 3. Elevation amphidromic system showing tidal range patterns $|2 S|$ (a) without and (b) with reference trench, which is indicated by a white rectangle. Co-phase lines are dashed, dividing the tidal period into 12 equal intervals. Panel (c) shows the differences due to the trench, with black lines representing the zerochange contours. Parameter values as in text; truncation number set at $M=80$.

current amphidromic charts without trench (figures 3a, 4a and 5a) effectively contain only a single compartment and thus extend the solution by Rienecker \& Teubner (1980) to account for cross-basin depth variations.

As shown by the elevation amphidromic system in figure 3, the reference trench causes changes in tidal range of the order of a few centimeters. As shown in figure 3c, these changes are felt roughly throughout the whole basin, alternatingly showing zones of decrease (blue) and zones of increase (red) in tidal range. Note that the largest change in coastal tidal range, over $3 \mathrm{~cm}$, occurs well away from the trench (as indicated by the circle at $x=B$ and $y \sim 500 \mathrm{~km}$ ). Furthermore, the changes at the opposite coast $x=0$, are of the order of $1 \mathrm{~cm}$. The pattern observed in figure $3 \mathrm{c}$ is associated with the different increases in wavelength experienced by the incoming and reflected Kelvin waves in C2, i.e. the middle compartment. For our reference trench, the wavelength of the incoming Kelvin wave increases from 750 to $753 \mathrm{~km}$, whereas the reflected Kelvin wave - which more strongly feels the presence of the trench - has a wavelength increasing more strongly from 683 to $699 \mathrm{~km}$. Although hardly visible from figures $3 \mathrm{a}$ and $\mathrm{b}$, the trench causes a shift of the EAPs over several $\mathrm{km}$ in the positive $y$-direction.

In contrast with the tidal range pattern, the effects of the trench on tidal currents are more strongly confined to the region of the trench (figures 4 and 5). At the trench center $\mathrm{Q}$, as introduced in figure $2 \mathrm{~d}$, the depth-averaged flow velocity amplitude in the along-basin direction increases by $2.1 \mathrm{~cm} / \mathrm{s}$, indicative of flow contraction (Roos et al. 2008). Flow contraction also explains the zone of increase immediately south and north of the trench. On the other hand, the cross-basin component at Q, dominated by continuity, decreases by $1.4 \mathrm{~cm} / \mathrm{s}$. In this reference case, the coastal velocity amplitudes change by less than $1 \mathrm{~cm} / \mathrm{s}$. 

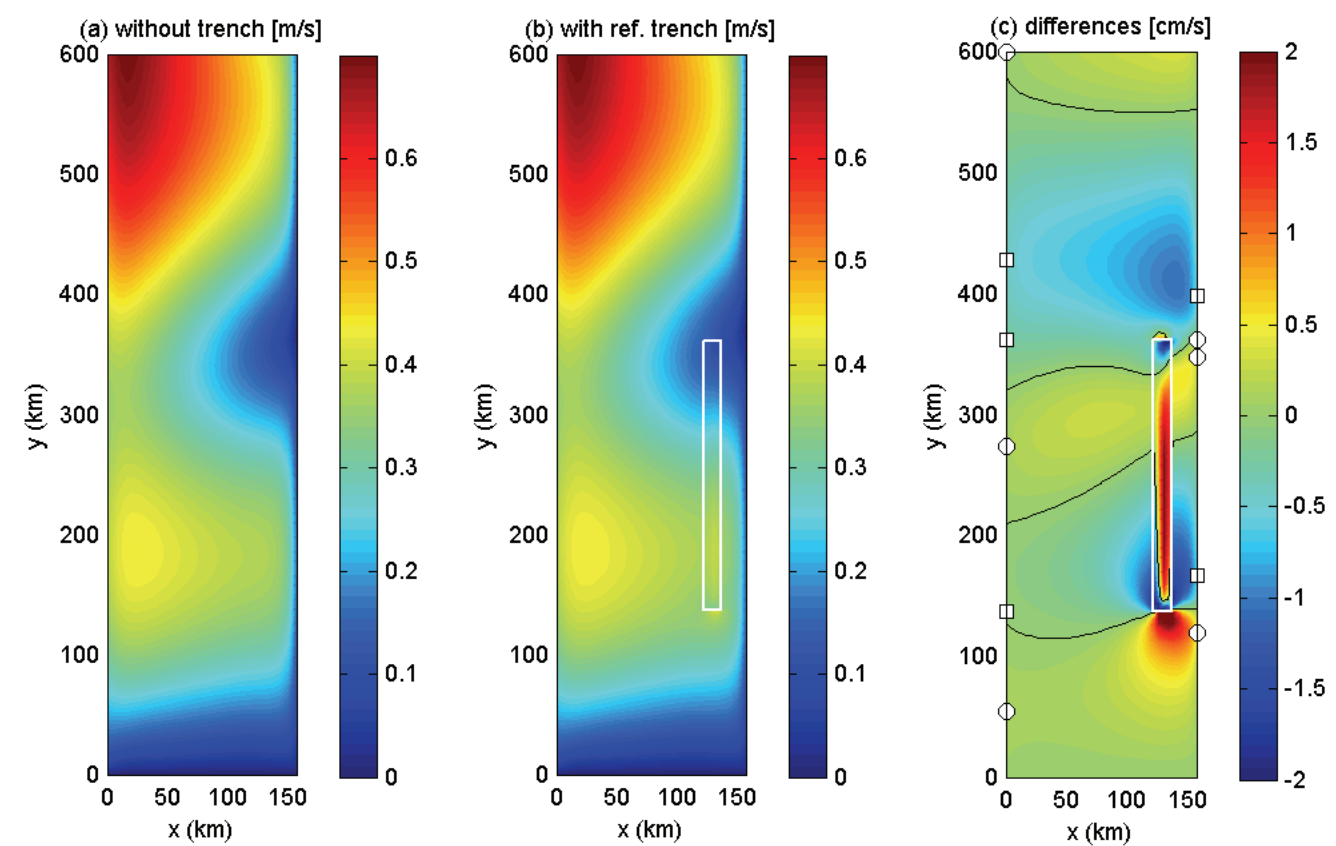

Figure 4. Same as figure 3, but now showing the amplitudes of the (depth-averaged) along-basin flow.
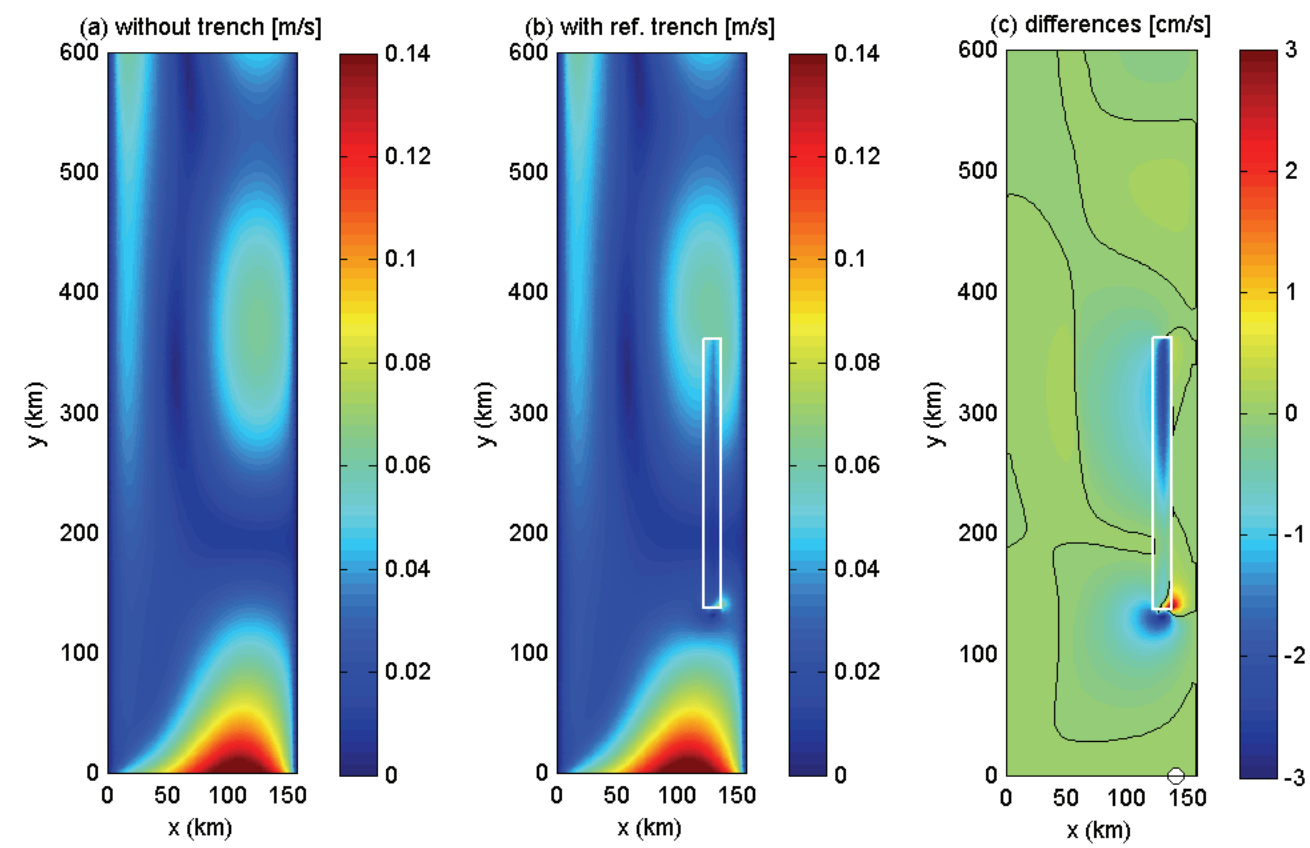

Figure 5. Same as figure 3, but now showing the amplitudes of the (depth-averaged) cross-basin flow.

\section{Influence of trench geometry}

To study the sensitivity of the model results to the trench dimensions, we have varied the dimensions in ranges around their default values, while fixing the along-basin trench centre at $y=250$ $\mathrm{km}$ and the rightmost boundary to NAP $-20 \mathrm{~m}$ depth contour. We have considered the following ranges in length, width and depth, implying variations in trench volume of 5.0-40.3 $\times 10^{9} \mathrm{~m}^{3}$ :

- $\quad$ Trench length: $L_{\mathrm{tr}}=112-448 \mathrm{~km}$

- Trench width: $B_{\mathrm{tr}}=6-21 \mathrm{~km}$

- Trench depth: $H_{\mathrm{tr}}=3-21 \mathrm{~m}$.

Without reporting the results in detail (presented in De Boer et al., submitted), the sensitivity analysis generally shows that the changes in tidal ranges and flow velocity amplitudes increase with 
increasing trench volume $V_{\text {tr }}$. Furthermore, the results are found to be more sensitive to changes in trench width and depth, than to changes in length. In particular, the deepest extraction scenario $\left(H_{\mathrm{tr}}=21\right.$ $\mathrm{m}$, other dimensions as in the reference case) leads to increases and decreases in coastal tidal range exceeding $5 \mathrm{~cm}$.

Again, the changes can be mainly explained by studying the interference pattern of the incoming and reflected Kelvin waves. As mentioned before, the properties of the reflected wave - which is bound to the coast $x=B$ nearest to the trench - are more sensitive to changes in trench dimensions than those of the incoming one. For example, the reflected Kelvin wavelength for the deepest extraction scenario equals $710 \mathrm{~km}$ (699 km for the reference case; $683 \mathrm{~km}$ without trench). The trench length does not affect these wavelengths, but it controls the distance over which the modified Kelvin wave manifests itself in the basin.

\section{CONCLUSIONS}

We have presented an idealized process-based modeling tool to assess the effects of a mega-scale sand extraction trench on tidal dynamics in semi-enclosed basins. This model has been applied to the typical long-term extraction scenario of a mega-scale trench of length $\sim 200 \mathrm{~km}$, width $\sim 10 \mathrm{~km}$ and depth $\sim 10$ $\mathrm{m}$, located in front of the Dutch coast.

The model results show that such an intervention affects the tidal range (several $\mathrm{cm}$ ), phase and currents (order $\mathrm{cm} / \mathrm{s}$ ), not only near but also tens to hundreds kilometers away from the extraction area. This highlights the difference between this mega-scale extraction scenario and current expertise with the more local effects of large-scale sandpits. Our results furthermore emphasize the importance of adopting a model domain on the scale of the basin, i.e. the Southern North Sea. Since our model does not include residual currents (M0) and overtides (M4, M6, ..), it is not geared to calculate sediment transport and resulting bed evolution. Nevertheless, the changes in tidal currents are likely to structurally alter sediment transport rates by several percents. This may affect coastal morphology, which is of concern to coastal managers.

Finally, idealized models may serve as supporting tool for more detailed, site-specific (and more time-consuming) simulations with a numerical engineering model. We argue that the added value is threefold. First, idealized models help to obtain insight in the physical mechanisms. Second, they may quickly provide the orders of magnitude of the effects and the area of influence, which helps e.g. to choose the domain of the engineering model. Finally, idealized models may serve as a quick tool for a study intro the effects of a wide variety of design alternatives.

\section{ACKNOWLEDGMENTS}

This research is supported by the Netherlands Technology Foundation STW, applied science division of NWO and the Technology Program of the Netherlands Ministry of Economic Affairs.

\section{REFERENCES}

De Boer, W. P., P. C. Roos, S. J. M. H. Hulscher, and A. Stolk. Impact of mega-scale sand extraction on tidal dynamics in semi-enclosed basins. An idealized model study with application to the Southern North Sea. Submitted.

Gill, A. E. (1982). Atmosphere-Ocean Dynamics, Academic Press, New York, 662 pp.

Rienecker, M. M., and M. D. Teubner (1980). A note on frictional effects in Taylor's problem, J. Mar. Res. 38(2), 183-191.

Roos, P. C., S. J. M. H. Hulscher, and H. J. de Vriend (2008). Modelling the morphodynamic impact of offshore sandpit geometries. Coast Eng. 55(9), 704-715. doi:10.1016/j.coastaleng.2008.02.019.

Roos, P. C., and H. M. Schuttelaars (2011). Influence of topography on tide propagation and amplification in semi-enclosed basins, Ocean Dyn.61(1), 21-38, doi:10.1007/s10236-010-0340-0.

Taylor, G. I. (1922). Tidal oscillations in gulfs and rectangular basins, Proc. Lond. Math. Soc. 20(1), 148-181, doi:10.1112/plms/s2-20.1.148.

Van der Veen, H. H., and S. J. M. H. Hulscher (2009). Predicting het occurrence of sand banks in the North Sea. Ocean Dyn. 59(5), 689-696. doi:10.1007/s10236-009-0204-7.

Van der Werf, J. J., A. Giardino, J. P. M. Mulder, and A. Stolk (2011). A first investigation into the impact of very large-scale offshore sand mining along the Dutch Coast, Proceedings Of The International Conference On Coastal Engineering, No. 32(2010), Shanghai, China. Paper \#: sediment.97. Retrieved from http://journals.tdl.org/ICCE/ 